Deans Ross (Orcid ID: 0000-0003-3831-2520)

Busch Florian (Orcid ID: 0000-0001-6912-0156)

\title{
Estimating stomatal and biochemical limitations during photosynthetic induction
}

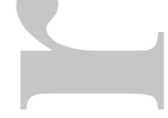

Running title: Limitations during photosynthetic induction

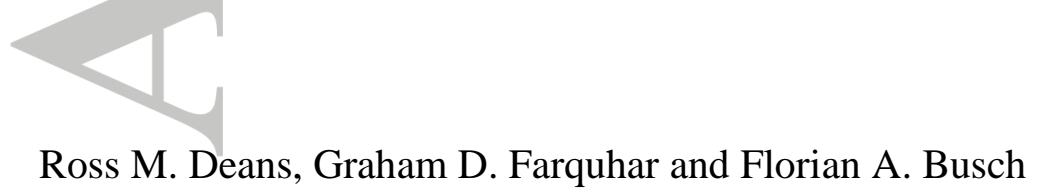

Ross M. Deans, Graham D. Farquhar and Florian A. Busch

ARC Centre of Excellence in Translational Photosynthesis, Division of Plant Science, Research School of Biology, The Australian National University, Canberra, ACT 2601, Australia

Corresponding author:

Florian A. Busch

Tel: +61261250123

Email: Florian.Busch@anu.edu.au

This article has been accepted for publication and undergone full peer review but has not been through the copyediting, typesetting, pagination and proofreading process which may lead to differences between this version and the Version of Record. Please cite this article as doi: $10.1111 /$ pce. 13622 
Summary statement: Methods that linearise stomatal and biochemical limitations during photosynthetic induction work best when stomatal opening and biochemical activation occur on similar timescales, while methods that sequentially remove limitations are preferred when stomatal opening occurs at a much slower rate than biochemical activation.

\section{Abstract}

Understanding stomatal and biochemical components that limit photosynthesis under different conditions is important for both the targeted improvement of photosynthesis and the elucidation of how stomata and biochemistry affect plant performance in an ecological context. Limitation analyses have not yet been extensively applied to conditions of photosynthetic induction after an increase in irradiance. Moreover, few studies have systematically assessed how well various limitation analyses actually work. Here we build on two general ways of estimating limitations, one that sequentially removes the effect of a limitation (elimination) and one that uses a tangent plane approximation (differential), by including the ternary effect and boundary layer conductance so that they are consistent with gas exchange data. We apply them to the analysis of temporal and time-integrated limitations during photosynthetic induction, calculating limitations either independent of the time course (one-step) or make use of the entire time course (stepwise). We show that the stepwise differential method is the best method to use when time steps are small enough. We further show that the differential method predicts limitations near exact when the internal $\mathrm{CO}_{2}$ concentration stays constant. This last insight has important implications for the general use of limitation analyses beyond photosynthetic induction. 
Keywords: photosynthetic induction, stomata, light, limitations, photosynthesis, kinetics of stomatal responses, biochemical activation

\section{Introduction}

Identifying photosynthetic limitations of a plant is integral to understanding the functioning of a plant in its environment. Limitation analyses address how various components limit photosynthesis (Jones, 1985). Not only can limitations provide insights into photosynthetic physiology under different conditions and in different genotypes, but they can also hint at where a plant should invest to gain the greatest benefit in photosynthesis. The major photosynthetic limitations at the leaf level can be broadly split into the restriction of $\mathrm{CO}_{2}$ supply to the site of carboxylation by diffusional effects and limitations in the consumption of $\mathrm{CO}_{2}$ by biochemical effects (Jones, 1973; Jones, 1985). Diffusional limitations can be further separated into stomatal effects limiting $\mathrm{CO}_{2}$ supply into the leaf, and mesophyll effects limiting $\mathrm{CO}_{2}$ supply from the intercellular airspace to the site of carboxylation (Grassi \& Magnani, 2005), while biochemical effects include the maximum rate of carboxylation by Rubisco and the capacity for regeneration of RuBP in the Calvin-Benson cycle (Busch \& Sage, 2017; Farquhar, von Caemmerer \& Berry, 1980).

Increasing the speed of photosynthetic induction under dynamic light environments has been suggested as a possible way to increase crop photosynthesis (Kaiser, Morales \& Harbinson, 2018; Kaiser et al., 2015; Kromdijk et al., 2016; Lawson \& Blatt, 2014; McAusland et al., 2016; Slattery, Walker, Weber \& Ort, 2018; Taylor \& Long, 2017). In response to an increase in irradiance, photosynthesis does not achieve its final value instantaneously but exhibits a delay. Thus, a leaf transiently forgoes potential photosynthesis, compared with the assimilation rate reaching steady state instantaneously (Deans, Brodribb, Busch \& Farquhar, 
2019). In a dynamic sense, a photosynthetic limitation at the leaf level can be defined as a reduction in the total photosynthesis achieved compared with that obtained if photosynthesis had reached its final value instantaneously, caused by both biochemical and diffusional effects (Fig. 1a). Firstly, photosynthesis is limited by the necessary build-up of Calvin-cycle intermediates and the slow induction of light-activated photosynthetic enzymes immediately following the increase in irradiance (Mott \& Woodrow, 2000). Build-up of the RuBP pool typically limits photosynthesis for approximately $60 \mathrm{~s}$ following an increase in irradiance, while full light activation of Rubisco typically takes 8-10 minutes (Mott \& Woodrow, 2000). Secondly, photosynthesis is limited by the supply of $\mathrm{CO}_{2}$ through slow stomatal opening (Kirschbaum \& Pearcy, 1988). Although some stomata respond rapidly following an increase in irradiance (e.g. in some monocot species), stomata can take much longer to fully open, potentially posing a significant photosynthetic limitation (Deans et al., 2019; McAusland et al., 2016; Vico, Manzoni, Palmroth \& Katul, 2011). Finally, mesophyll conductance has been shown to increase with irradiance (Douthe, Dreyer, Brendel \& Warren, 2012; Douthe, Dreyer, Epron \& Warren, 2011; Flexas, Ribas - Carbo, Diaz - Espejo, Galmes \& Medrano, 2008). These changes in mesophyll conductance occur rapidly, in the order of minutes (Douthe et al., 2011). However, changes in mesophyll conductance with changing irradiance are not universal across species (Tazoe, von Caemmerer, Badger \& Evans, 2009). Variable mesophyll conductance potentially poses another significant limitation to photosynthesis in dynamic light environments.

Despite the overall effects of photosynthetic limitations being clearly apparent during photosynthetic induction, few studies have utilised photosynthetic limitation analyses to quantify the contributions of biochemical and diffusional limitations in this setting (Taylor \& Long, 2017). Limitation analyses also typically utilise simplified equations that ignore the ternary correction and boundary layer conductance (von Caemmerer \& Farquhar, 1981). The 
ternary effect takes into account the diffusion of $\mathrm{CO}_{2}$ in water vapour (Jarman, 1974), while the boundary layer conductance accounts for the extra resistance of the boundary layer in the diffusion of gas from the leaf surface to the surroundings. Although these effects are normally only significant at high transpiration rates and stomatal conductances, respectively, gas exchange data are typically calculated including both effects. Here we derived equations for two methods to calculate limitations including these effects and applied them during photosynthetic induction. The first, an elimination method, calculates limitations by removing the effect of each limitation sequentially (Assmann, 1988; Jones, 1985; Wilson, Baldocchi \& Hanson, 2000). The second, a differential method, allocates limitations by linearising the impact of each limitation on assimilation rate (Grassi \& Magnani, 2005; Jones, 1985; Wilson et al., 2000). We estimated both the temporal dynamics of absolute limitations, as well as time-integrated relative photosynthetic limitations during photosynthetic induction, on a set of 42 such inductions from Deans et al. (2019), comprising 15 species. We did this for both methods in two different ways: a step-wise method that took into account previous and subsequent limitation estimates, and a one-step method that did not. Our aim was to systematically compare the performance of the different ways of estimating limitations during photosynthetic induction, and to determine what method is best to use and when.

\section{Theory}

As described in Deans et al. (2019), following an increase in irradiance from low light or darkness, slow biochemical activation and stomatal opening limits the rate of photosynthesis the plant can achieve compared with the final steady-state photosynthetic rate. This instantaneous difference in photosynthetic rate $(d A)$ is defined as 
$d A(t)=A_{f}-A(t)$

where $A_{\mathrm{f}}$ is the final assimilation rate at steady state and $A(t)$ is the current assimilation rate at time $t$.

It is also interesting to know how much total assimilation the plant forgoes during photosynthetic induction. Forgone assimilation $(\Delta C)$ is defined as

$\Delta C=\int_{0}^{t_{f}} d A(t) d t=\int_{0}^{t_{f}}\left(A_{f}-A(t)\right) d t$,

where $t_{\mathrm{f}}$ is the time corresponding to $A_{\mathrm{f}}$ and the change in irradiance occurs at $t=0$.

The aim of the limitation analyses here is to separate $d A$ and $\Delta C$ into stomatal and biochemical components. To do so requires the calculation of limitation estimates on $d A$ at each time point. There are two ways that this can be done. The first, here called a one-step method, calculates the limitation from the final $A$ directly (Fig. 1a). It is computationally simpler, but large errors may be expected if the final $A$ state is far from the current state. This approach is closer to state function approaches for the estimate of limitations, because the current limitation of $d A$ is calculated without knowledge of the limitations affecting neighbouring time points (Jones, 1985). The second, here called a stepwise method, calculates the limitation to the next time point, and then the limitation of $d A$ is calculated by summing all the limitation components from the final time point back to the current one (Fig. 1b). This method is computationally more demanding, but error in limitation estimates may be expected to be smaller provided time steps between points is small enough. This method is a path-dependent method because knowledge of limitations throughout the time course of induction is used to calculate the current limitation (Jones, 1985).

Calculation of biochemical and stomatal limitation during photosynthetic induction require an expression for $A$ in terms of the component limitations. Biochemical limitations during photosynthetic induction are predominantly caused by Rubisco activation, with RuBP regeneration only limiting for less than one minute after an increase in irradiance (Mott \& 
Woodrow, 2000). It is instructive then to consider $A$ as a function of the maximum carboxylation rate of Rubisco in the current activation state $\left(V_{\mathrm{cmax}}\right)$ and stomatal conductance to $\mathrm{CO}_{2}\left(g_{\mathrm{sc}}\right)$; that is,

$A(t) \equiv A\left(V_{c \max }(t), g_{s c}(t)\right)$

We note here that $g_{\mathrm{sc}}=g_{\mathrm{sw}} / 1.6$, where $g_{\mathrm{sw}}$ is the stomatal conductance to water vapour, the more commonly calculated stomatal conductance in gas exchange systems. The factor 1.6 takes into account the ratio of the diffusivities of water and $\mathrm{CO}_{2}$ in air.

An expression for $A$ is required in terms of $V_{\mathrm{cmax}}$ and $g_{\mathrm{sc}}$. The Rubisco-limited biochemistry of photosynthesis can be described by (Farquhar et al., 1980)

$A=\frac{V_{c \max }\left(C_{i}-\Gamma^{*}\right)}{C_{i}+K_{m}}-R_{d}$,

where $C_{\mathrm{i}}$ is the $\mathrm{CO}_{2}$ concentration in the intercellular airspace, $\Gamma^{*}$ is the $\mathrm{CO}_{2}$ compensation point in the absence of respiration, $K_{\mathrm{m}}$ is the effective Michaelis-Menten constant taking into account the $\mathrm{O}_{2}$ concentration and $R_{\mathrm{d}}$ is the rate of mitochondrial respiration in the light. Here it was assumed that the $\mathrm{CO}_{2}$ concentration at the site of carboxylation was equal to $C_{\mathrm{i}}$ because steady state and dynamic mesophyll conductance were not consistently known in the plants sampled.

An equation for $C_{\mathrm{i}}$ is required. From von Caemmerer and Farquhar (1981), $C_{\mathrm{i}}$ including the ternary correction is

$C_{i}=\frac{C_{a}\left(g_{t c}-\frac{E}{2}\right)-A}{\left(g_{t c}+\frac{E}{2}\right)}$,

where $C_{\mathrm{a}}$ is the ambient $\mathrm{CO}_{2}$ concentration and $g_{\mathrm{tc}}$ is the total conductance to $\mathrm{CO}_{2}$ from outside the leaf boundary layer to inside the leaf. $g_{\text {tc }}$ can be further broken up into

$g_{t c}=\frac{g_{s c} g_{b c}}{g_{s c}+g_{b c}}$,

where $g_{\mathrm{bc}}$ is the boundary layer conductance to $\mathrm{CO}_{2}$.

Substituting Eqn 4 into Eqn 3 and rearranging gives 


$$
\begin{aligned}
& A^{2}-\left[V_{c m a x}-R_{d}+C_{a}\left(g_{t c}-\frac{E}{2}\right)+K_{m}\left(g_{t c}+\frac{E}{2}\right)\right] A+V_{c m a x}\left[C_{a}\left(g_{t c}-\frac{E}{2}\right)-\Gamma^{*}\left(g_{t c}+\right.\right. \\
& \left.\left.\frac{E}{2}\right)\right]-R_{d}\left[C_{a}\left(g_{t c}-\frac{E}{2}\right)+K_{m}\left(g_{t c}+\frac{E}{2}\right)\right]=0 .
\end{aligned}
$$

$A$ thus has the solution

$$
A=\left(\frac{1}{2 a}\right)\left(b-\sqrt{b^{2}-4 a c}\right)
$$

where

$a=1$

$b=V_{c \max }-R_{d}+C_{a}\left(g_{t c}-\frac{E}{2}\right)+K_{m}\left(g_{t c}+\frac{E}{2}\right)$

and

$c=V_{c \max }\left[C_{a}\left(g_{t c}-\frac{E}{2}\right)-\Gamma^{*}\left(g_{t c}+\frac{E}{2}\right)\right]-R_{d}\left[C_{a}\left(g_{t c}-\frac{E}{2}\right)+K_{m}\left(g_{t c}+\frac{E}{2}\right)\right]$.

(Eqn 7c)

The two methods to calculate limitations will rely on Eqns 3, 5, 6 and 7 in both the derivations and calculations of limitations.

\section{Elimination methods}

Elimination methods sequentially remove the effect of one limitation and calculate the resulting difference in assimilation, until all limitations have been accounted for (Jones, 1973; Jones, 1985). However, the nonlinear nature of photosynthesis means that there is often not a unique solution, and that a different solution will be obtained depending on the order that the limitation effects are removed (Fig. 1b). It is for this reason that it is referred to as a pathdependent method (Jones, 1973). Considering dynamic changes in $V_{\text {cmax }}, g_{\text {sc }}$ and $E$ only, and if $V_{\mathrm{cmax} 1}, g_{\mathrm{sc} 1}$ and $E_{1}$, and $V_{\mathrm{cmax} 2}, g_{\mathrm{sc} 2}$ and $E_{2}$ are the of $V_{\mathrm{cmax}}, g_{\mathrm{sc}}$ and $E$ at two photosynthetic states, respectively, then there are two ways of calculating the respective limitations:

(1) Removing the stomatal limitation first gives 
$\delta A_{\text {stom,stom }}=A\left(V_{c \max 1}, g_{s c 2}, E_{2}\right)-A\left(V_{c \max 1}, g_{s c 1}, E_{1}\right)$

(Eqn 8)

and

$\delta A_{\text {biochem, stom }}=A\left(V_{\text {cmax } 2}, g_{s c 2}, E_{2}\right)-A\left(V_{c \max 1}, g_{s c 2}, E_{2}\right)$

where the first subscript of $\delta A$ indicates the limitation and the second indicates which limitation was eliminated first.

(2) Removing the biochemical limitation first gives

$\delta A_{\text {biochem }, \text { biochem }}=A\left(V_{c m a x 2}, g_{s c 1}, E_{1}\right)-A\left(V_{c m a x 1}, g_{s c 1}, E_{1}\right)$

and

$\delta A_{\text {stom,biochem }}=A\left(V_{\text {cmax } 2}, g_{s c 2}, E_{2}\right)-A\left(V_{c \max 2}, g_{s c 1}, E_{2}\right)$

The intermediate assimilation rates are calculated using Eqn 7, substituting in the relevant values for $V_{\mathrm{cmax}}, g_{\mathrm{sc}}$ and $E$ as required. A graphical example of the two elimination approaches is illustrated in Fig. 1c.

Using the one-step method of calculating limitations, state 1 is the current time point and state 2 is the final time point and

$d A_{x, y}(t)=\delta A_{x, y}(t)$

(Eqn 12)

where $\mathrm{x}$ is the limitation and $\mathrm{y}$ is the limitation that was removed first.

Using the stepwise method, state 1 is the current time point and state 2 is the next time point. $d A_{\mathrm{x}, \mathrm{y}}$ for the current time point is then calculated as

$d A_{x, y}(t)=\sum_{i=t}^{t_{f}} \delta A_{x, y}(i)$

where $i$ is a dummy variable for $t$.

The time-integrated relative limitation over the duration of photosynthetic induction $\left(\sigma_{\mathrm{x}, \mathrm{y}}\right)$ was calculated by integrating the calculated $d A_{\mathrm{x}, \mathrm{y}}$ over the time course and dividing by forgone assimilation, $\Delta C$; that is

$\sigma_{x, y}=\frac{1}{\Delta C} \int_{0}^{t_{f}} d A_{x, y}(t) d t$, 
where the integration is again done from the time of change in irradiance $(t=0)$ to time $t=t_{\mathrm{f}}$, the time corresponding to the final assimilation rate $A_{\mathrm{f}}$.

Typically limitations should range between 0 and 1; e.g. a $\sigma_{\text {stom,biochem }}$ of 0.4 indicates $40 \%$ of the forgone photosynthesis during photosynthetic induction was due to slow stomatal opening, while $60 \%$ was due to slow biochemical activation, when evaluated removing the biochemical limitation first. However, values for limitations $<0$ or $>1$ are possible, and represent occasions where one or both $g_{\mathrm{sc}}$ or $V_{\mathrm{cmax}}$ are transiently greater than their final values at some point during photosynthetic induction. For example, if stomata transiently overshoot the final $g_{\mathrm{sc}}$ value, then stomata become less limiting than if $g_{\mathrm{sc}}$ was at the final steady state value, and the value for, say, $d A_{\text {stom,biochem }}$ would become negative. If the

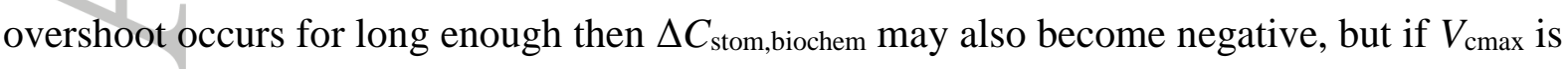
rising monotonically to its final value then $\Delta C_{\text {biochem,biochem }}$ would be positive and greater than $\Delta C$ by definition. Thus $\sigma_{\text {stom,biochem }}$ would be $<0$ and $\sigma_{\text {biochem,biochem }}$ would be $>1$. In this scenario, if $\Delta C$ is small compared to the component limitations, poorer estimates of timeintegrated relative limitations may be expected.

From the elimination method we obtain two potentially different estimates of limitations, depending on which limitation is removed first. It is instructive then to consider the mean and a range estimate of the limitations calculated. The mean component of $d A$ due to a limitation $\mathrm{x}$ (i.e. stomatal or biochemical) at each time point $\left(d A_{\mathrm{x}, \mathrm{mean}}\right)$ was defined as

$$
d A_{x, \text { mean }}=\frac{1}{2}\left(d A_{x, \text { stom }}+d A_{x, \text { biochem }}\right) .
$$

The mean time-integrated limitation $\left(\sigma_{\mathrm{x}, \text { mean }}\right)$ is then

$$
\sigma_{x, \text { mean }}=\frac{1}{2}\left(\sigma_{x, \text { stom }}+\sigma_{x, \text { biochem }}\right)
$$

The relative range in $d A$ for the elimination method ( $r e l$. range $(d A)_{\text {elim }}$ ) was defined as the absolute value of the difference between the two estimates of a limitation, divided by the instantaneous difference in photosynthetic rate $(d A)$; i.e. 
rel.range $(d A)_{\text {elim }}=\frac{\left|d A_{x, y}-d A_{x, x}\right|}{|d A|}$,

where $\mathrm{x}$ and $\mathrm{y}$ are limitations (i.e. stomatal or biochemical). Similarly the relative range in $\Delta C$ (rel. range $\left.(\Delta C)_{\text {elim }}\right)$ can be defined as

rel.range $(\Delta C)_{\text {elim }}=\frac{\left|\Delta C_{x, y}-\Delta C_{x, x}\right|}{|\Delta C|}$

where

$\Delta C_{x, y}=\int_{0}^{t_{f}} d A_{x, y}(t) d t$

It is shown in the Supporting Information that the relative range estimates are identical whether the range is calculated using the stomatal or biochemical limitations.

\section{Differential method}

The differential method uses a linear approximation to calculate the effect of limitations on photosynthesis (Deans et al., 2019; Grassi \& Magnani, 2005; Jones, 1973; Jones, 1985; Wilson et al., 2000). Ignoring the ternary effect for now, we can consider $A\left(V_{\mathrm{cmax}}, g_{\mathrm{sc}}\right)$ as an "assimilation surface", and $A_{1}=A\left(V_{\mathrm{cmax} 1}, g_{\mathrm{sc} 1}\right)$ and $A_{2}=A\left(V_{\mathrm{cmax} 2}, g_{\mathrm{sc} 2}\right)$, as two points on the surface (Fig. 1d). $A(t)$ tracks a path on the "assimilation surface" through time as photosynthetic induction occurs. Because $A$ is a nonlinear surface we cannot directly calculate the component that each limitation contributes to $\delta A$; however, $\delta A$ can be linearised using a Taylor series. Below we derive the differential method including both the ternary correction and boundary layer conductance.

Following Deans et al. (2019), $\delta A$ can be linearised as

$$
\delta A=\frac{\partial A}{\partial V_{c \max }} \delta V_{c \max }+\frac{\partial A}{\partial g_{s c}} \delta g_{s c}+\text { error }
$$

where the partial derivatives are evaluated at state 1 and $\delta V_{\mathrm{cmax}}$, and $\delta g_{\mathrm{sc}}$ are the differences in the respective variables between state 2 and state 1 . We can depict this linearisation as a 
tangent plane that touches the "assimilation surface" at the current state (Fig. 1d). Individual differences in assimilation can be identified as

$$
\begin{aligned}
& \delta A_{\text {biochem }}=\frac{\partial A}{\partial V_{c \max }} \delta V_{c \max } \\
& \text { and } \\
& \delta A_{\text {stom }}=\frac{\partial A}{\partial g_{s c}} \delta g_{s c},
\end{aligned}
$$

where $\delta A_{\text {biochem }}$ and $\delta A_{\text {stom }}$ are the components of $\delta A$ due to biochemical and stomatal limitations, respectively.

It is noted here that the single-point calculation of limitations using a control coefficient or sensitivity analysis is a special case of the two-point limitation analysis, where one point is taken as zero (Jones, 1985; Woodrow \& Berry, 1988); that is

$$
S_{V c \max }=\frac{\partial A}{\partial V_{c \max }} \frac{V_{c \max }}{A}
$$

and

$$
S_{g s c}=\frac{\partial A}{\partial g_{s c}} \frac{g_{s c}}{A}
$$

where $S_{\mathrm{V} \text { cmax }}$ and $s_{\mathrm{gsc}}$ are the sensitivity or control coefficient for respective limitations.

The calculation of each limitation requires the evaluation of the partial derivatives of $A$ with respect to $V_{\text {ćmax }}$ and $g_{\text {sc. }}$ We appeal to Eqn 6, calculating derivatives implicitly. Differentiating Eqn 6 with respect to $V_{\text {cmax }}$ and substituting into Eqn 21 gives

$$
\delta A_{\text {biochem }}=\frac{C_{a}\left(g_{t c}-\frac{E}{2}\right)-\Gamma^{*}\left(g_{t c}+\frac{E}{2}\right)-A}{\left[V_{c \max }-R_{d}+C_{a}\left(g_{t c}-\frac{E}{2}\right)+K_{m}\left(g_{t c}+\frac{E}{2}\right)-2 A\right]} \delta V_{c m a x} .
$$

Differentiating Eqn 6 with respect to $g_{\text {sc }}$ is more complicated, as altering $g_{\text {sc }}$ will have an effect on $E$. The ternary-corrected equation for $E$ is

$$
E=\frac{g_{t w}\left(w_{i}-w_{a}\right)}{\left[1-\frac{\left(w_{i}+w_{a}\right)}{2}\right]},
$$


(von Caemmerer \& Farquhar, 1981) where $g_{\text {tw }}$ is the total conductance to water vapour, and $w_{\mathrm{i}}$ and $w_{\mathrm{a}}$ are the water mole fractions inside and outside the leaf, respectively. $g_{\mathrm{tw}}$ can be expressed as

$g_{t w}=\frac{g_{s w} g_{b w}}{g_{s w}+g_{b w}}=\frac{(1.6) g_{s c}(1.37) g_{b c}}{1.6 g_{s c}+1.37 g_{b c}}$

where $g_{\mathrm{sw}}$ and $g_{\mathrm{bw}}$ are the stomatal and boundary layer conductances to water vapour, respectively. The factor 1.37 interconverting the boundary layer conductances to water vapour and $\mathrm{CO}_{2}$ comes from a consideration of mass flow in laminar flows (von Caemmerer \& Farquhar, 1981).

Further chamber feedbacks of $E$ on $w_{\mathrm{a}}$ are ignored. It can be shown that

$\frac{\partial\left(g_{t c} \pm \frac{E}{2}\right)}{\partial g_{s c}}=\frac{1}{g_{s c}^{2}}\left(g_{t c}^{2} \pm \frac{g_{t w}}{1.6} \frac{E}{2}\right)$,

so that

$\delta A_{\text {stom }}=\left(\frac{1}{g_{s c}^{2}}\right) \frac{C_{a}\left[V_{c m a x}-\left(A+R_{d}\right)\right]\left(g_{t c}^{2}-\frac{g_{t w} E}{1.62}\right)-\left[V_{c m a x} \Gamma^{*}+K_{m}\left(A+R_{d}\right)\right]\left(g_{t c}^{2}+\frac{g_{t w} E}{1.62}\right)}{\left[V_{c m a x}-R_{d}+C_{a}\left(g_{t c}-\frac{E}{2}\right)+K_{m}\left(g_{t c}+\frac{E}{2}\right)-2 A\right]} \delta g_{s c}$.

The components of $\delta A$ due to each limitation are calculated using Eqns 23 and 27. For the one-step calculation of limitations, state 1 is the current time point and state 2 is the final time point and

$d A_{x}(t)=\delta A_{x}(t)$

where $\mathrm{x}$ is the limitation.

For the stepwise calculation of limitations, state 1 is the current time point and state 2 is the next time point. $d A_{\mathrm{x}}$ is then calculated as

$d A_{x}(t)=\sum_{i=t}^{t_{f}} \delta A_{x}(i)$

where $i$ is again a dummy variable for $t$.

Neither Wilson et al. (2000) nor Grassi and Magnani (2005) consider the effect of boundary layer conductance or of the ternary correction. They also follow a different route for splitting 
the effect of limitations, initially noting that changing either $C_{\mathrm{i}}$ or $V_{\mathrm{cmax}}$ can change assimilation and then further noting that a change in $C_{\mathrm{i}}$ can occur by changing $A$ or $g_{\mathrm{sc}}$. If the ternary correction and boundary layer conductance are ignored in our analysis (i.e. $g_{\mathrm{bc}}, g_{\mathrm{bw}}$ and $E$ all set to zero), the two approaches used to derive limitations are identical (see Supporting Information).

The total assimilation forgone due to a limitation $\mathrm{x}$ (either biochemical or stomatal) over the duration of photosynthetic induction can be calculated as

$$
\Delta C_{x}=\int_{0}^{t_{f}} d A_{x} d t
$$

The instantaneous difference in photosynthetic rate estimated by the differential method $\left(d A_{\text {diff }}\right)$ is

$$
d A_{\text {diff }}=d A_{\text {biochem }}+d A_{\text {stom }}
$$

while the estimate of forgone assimilation $\left(\Delta C_{\text {diff }}\right)$ is

$$
\Delta C_{\text {diff }}=\Delta C_{\text {biochem }}+\Delta C_{\text {stom }}
$$

The time-integrated limitation over the duration of photosynthetic induction $\left(\sigma_{\mathrm{x}}\right)$ is then (Deans et al., 2019)

$$
\sigma_{x}=\frac{\Delta C_{x}}{\Delta C_{\text {diff }}}
$$

where $\mathrm{x}$ is the limitation (that is, biochemical or stomatal).

Unlike the elimination method, there is only one estimate for the effect of each limitation on forgone potential assimilation using the differential method. Instead linearisation introduces an intrinsic error in $d A_{\text {diff }}$ and $\Delta C_{\text {diff, }}$, causing them to differ from $d A$ and $\Delta C$, respectively. We can define the relative error in $d A$ for the differential method (rel. error $\left.(d A)_{\text {diff }}\right)$ as

$$
\text { rel.error }(d A)_{\text {diff }}=\frac{\left|d A-d A_{d i f f}\right|}{|d A|} \text {. }
$$

Likewise for $\Delta C$ we can define the relative error $\left(\operatorname{rel}\right.$. $\left.\operatorname{error}(\Delta C)_{\mathrm{diff}}\right)$ as

$$
\text { rel.error }(\Delta C)_{\text {diff }}=\frac{\left|\Delta C-\Delta C_{d i f f}\right|}{|\Delta C|}
$$




\section{Methods}

Experimental testing of limitations

The elimination and differential methods of estimating limitations were tested on a data set of 42 time courses of photosynthetic induction following an increase in irradiance from 15 species, from the study of Deans et al. (2019). Species tested were Adiantum capillus-veneris, Austrocedrus chilensis, Callitris preissii, Dicksonia antarctica, Eucalyptus pauciflora, Gossypium hirsutum, Histiopteris incisa, Hypolepis rugolusa, Marsilea drummondii, Nothofagus cunninghamii, Pinus radiata, Prumnopitys ladei, Spinacea oleracea cv. 'Viking', Vicia faba and Vitis vinifera cv. 'Chardonnay' and represent a broad range of stomatal opening times and photosynthetic capacities.

In Deans et al. (2019) gas exchange dynamics were measured using an infrared gas analyser (IRGA; LI-6400XT; LICOR Biosciences, Lincoln, Nebraska, USA), maintaining reference $\mathrm{CO}_{2}$ concentration and chamber temperature. This meant that $C_{\mathrm{a}}$ in the chamber decreased during photosynthetic induction. For both calculating $C_{\mathrm{i}}$ and in further calculations of limitations at each time point, the $C_{\mathrm{a}}$ at that point in time was used. The response time for the chamber was also ignored, although the small chamber volume relative to leaf area and system flow rate, the IRGAs being close to the site of gas exchange in this system, and the changes in chamber conditions occurring via plant responses and not step changes in incoming gas composition together mean that the effect is small.

All methods for calculating limitations require an estimate of how $V_{\text {cmax }}$ changes throughout photosynthetic induction. $V_{\text {cmax }}$ was obtained by rearranging Eqn 3 and solving for $V_{\text {cmax }}$ :

$$
V_{c \max }(t)=\frac{\left(A(t)+R_{d}\right)\left(C_{i}(t)+K_{m}\right)}{C_{i}(t)-\Gamma^{*}}
$$


$R_{\mathrm{d}}$ was assumed to be the same as dark respiration, while $\Gamma^{*}$ and $K_{\mathrm{m}}$ were taken as the mean values for $\mathrm{C}_{3}$ species from Hermida-Carrera, Kapralov and Galmés (2016) as 41.2 and 529.4 $\mu \mathrm{mol} \mathrm{mol}{ }^{-1}$, respectively, at $25^{\circ} \mathrm{C}$.

Stomatal and biochemical components of $d A$ were estimated at each time point throughout time courses of photosynthetic induction for all 42 traces, comparing the elimination methods (Eqns 8-11) with the differential method (Eqns 23 and 27) using both the one-step (Eqns 12 and 28) and stepwise calculation of limitations (Eqns 13 and 29). This required the calculation of $g_{\text {tc }}$ and $g_{\text {tw }}$ at each time point using Eqns 5 and 25, respectively. $g_{\text {bw }}$ was assumed to be $2.84 \mathrm{~mol} \mathrm{~m}^{-2} \mathrm{~s}^{-1}$, the value for the $6 \mathrm{~cm}^{2}$ chamber with fast fan speed for the LI-6400XT. $d A_{\text {diff }}$ was also calculated at each time point for both the one-step and stepwise methods (Eqn 31)

Components of $\Delta C$ due to each limitation were calculated for the elimination methods $\left(\Delta C_{\mathrm{x}, \mathrm{y}}\right.$, Eqn 19), as was observed forgone assimilation ( $\Delta C$, Eqn 2). These were used to calculate time-integrated relative limitations during photosynthetic induction for the elimination methods $\left(\sigma_{\mathrm{x}, \mathrm{y}}\right.$, Eqn 14) and the relative range of the elimination methods $\left(\mathrm{rel}\right.$. $\operatorname{range}(\Delta C)_{\mathrm{elim}}$, Eqn 18). Components of $\Delta C$ due to each limitation were calculated for the differential method $\left(\Delta C_{\mathrm{x}}\right.$, Eqn 30), along with the total forgone assimilation predicted by the differential method $\left(\Delta C_{\text {diff, }}\right.$ Eqn 32). These were used to calculate the time-integrated relative limitations during photosynthetic induction for the differential method $\left(\sigma_{\mathrm{x}}\right.$, Eqn 33) and the relative error of the differential method $\left(\operatorname{rel}\right.$. $\operatorname{error}(\Delta C)_{\mathrm{diff}}$ Eqn 35).

Stomatal opening $\left(t_{90 \mathrm{~g}}\right)$ and biochemical activation ( $\left.\mathrm{t}_{90 \mathrm{v}}\right)$ response times were defined as the time to $90 \%$ of the difference between the respective initial and maximum values of $g_{\mathrm{sc}}$ and $V_{\text {cmax }}$, following Deans et al. (2019). 
Simulating the relative effect of stomatal opening and biochemical activation times on error in the limitation analyses

Photosynthetic induction following an increase in irradiance was modelled for a range of stomatal opening and biochemical activation times to investigate the effect of each on the relative range of the elimination method and the relative error of the differential method. The increase from low to high irradiance was taken to occur at time $t=0 \mathrm{~min}$. For simplicity, biochemical activation and stomatal opening were assumed to follow exponential rise to maximum kinetics in simulations, despite variation in the dynamic behaviour of photosynthetic induction; i.e. for biochemical activation

$$
V_{c \max }(t)=V_{c \max f}+\left(V_{c \operatorname{maxi}}-V_{c \max f}\right) e^{-\frac{t}{\tau_{V}}},
$$

where $V_{\text {cmaxi }}$ and $V_{\text {cmaxf }}$ are the initial and final $V_{\mathrm{cmax}}$, respectively, and $\tau_{\mathrm{V}}$ is the characteristic time for biochemical activation; and for stomatal opening

$g_{s c}(t)=g_{s c f}+\left(g_{s c i}-g_{s c f}\right) e^{-\frac{t}{\tau_{g}}}$

where $g_{\text {sci }}$ and $g_{\text {scf }}$ are the initial and final $g_{\text {sc }}$, respectively, and $\tau_{\mathrm{g}}$ is the characteristic time for stomatal opening. $t_{90}$ and $\tau$ for both stomatal opening and biochemical activation were interrelated by

$t_{90}=-\tau \log _{e}(0.1)$

At each time point $E$ was calculated from Eqn 24, assuming $w_{\mathrm{i}}$ and $w_{\mathrm{a}}$ of 0.032 and $0.022 \mathrm{~mol}$ $\mathrm{mol}^{-1}$, respectively, giving a leaf-to-air vapour pressure difference of approximately $1 \mathrm{kPa}$ at $25^{\circ} \mathrm{C}$. A was calculated using Eqn 7.

Time courses of photosynthetic induction were simulated using one set of steady-state photosynthetic parameters. Initial and final steady-state values for $V_{\mathrm{cmax}}$ used were $V_{\mathrm{cmaxi}}=10$ $\mu \mathrm{mol} \mathrm{m} \mathrm{m}^{-2} \mathrm{~s}^{-1}$ and $V_{\text {cmaxf }}=80 \mu \mathrm{mol} \mathrm{m} \mathrm{m}^{-2} \mathrm{~s}^{-1}$, respectively, while $g_{\text {sci }}$ and $g_{\text {scf }}$ were scaled to maintain the same $g_{\mathrm{sc}} / V_{\mathrm{cmax}}$ ratio at low and high irradiance, akin to maintaining constant $C_{\mathrm{i}}$ 
at the two steady states (Wong, Cowan \& Farquhar, 1979). The $g_{\mathrm{sc}} / V_{\mathrm{cmax}}$ ratio used was $2.8 \mathrm{x}$ $10^{3}$ and was the mean ratio of $g_{\text {scf }}$ and $V_{\text {cmaxf }}$ observed across the data from Deans et al. (2019). $R_{\mathrm{d}}$ was set at zero for simplicity. $\tau_{\mathrm{V}}$ was set at $5 \mathrm{~min}$, while simulations encompassed values of $\log _{10}\left(\tau_{\mathrm{g}} / \tau_{\mathrm{V}}\right)$ between -0.75 and 1.45. Simulated time courses were run for $600 \mathrm{~min}$. $d A(t)$ and $d A_{\text {diff }}(t)$ were calculated for each simulation, while the relative range for the

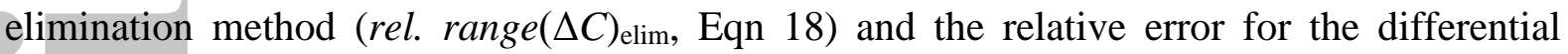

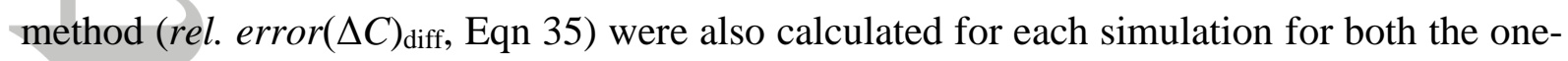
step and stepwise methods.

The effect of boundary layer conductance, ternary correction and consistency in gas exchange equations on limitation estimates

The equations used in most gas exchange systems include both boundary layer conductance and ternary corrections (von Caemmerer \& Farquhar, 1981), while the gas exchange equations employed in limitation analyses typically ignore their effects (Grassi \& Magnani, 2005; Wilson et al., 2000). We wanted to test: (1) whether boundary layer conductance and ternary correction have a large impact on the estimation of limitations when the equations used in calculating gas exchange data and limitation analyses are consistent? (2) what effect does using gas exchange data and limitation analyses with different assumptions have on the estimation of limitations? To test both questions, simpler forms of the limitation analyses were performed, ignoring either the ternary correction $(E=0$ in analysis equations) or both the ternary correction and boundary layer conductance $\left(E=0, g_{\mathrm{tc}}=g_{\mathrm{sc}}\right.$ and $g_{\mathrm{tw}}=g_{\mathrm{sw}}$ in analysis equations). These were tested on gas exchange data that were recalculated to match the assumptions used in the analysis (consistent), or on the full ternary and boundary layer gas exchange data as are usually obtained from gas exchange systems. For the case of 
ignoring the ternary correction but including boundary layer conductance, total conductance to water vapour was calculated at each time point as

$$
g_{t w}=\frac{E}{\left(V P D L / P_{a t m}\right)} \text {, }
$$

where $V P D L$ is the leaf-to-air vapour pressure difference and $P_{\text {atm }}$ is atmospheric pressure, assumed to be $100 \mathrm{kPa}$.

Stomatal conductance to $\mathrm{CO}_{2}\left(g_{\text {sc }}\right)$ was then recalculated as

$$
g_{s c}=\frac{g_{b w} g_{t w}}{1.6\left(g_{b w}-g_{t w}\right)}
$$

and total conductance to $\mathrm{CO}_{2}\left(g_{\mathrm{tc}}\right)$ calculated using Eqn 5. $C_{\mathrm{i}}$ was then recalculated at each time point as

$$
C_{i}=C_{a}-\frac{A}{g_{t c}}
$$

For the case of ignoring both the ternary correction and boundary layer conductance, total conductance to water vapour was calculated at each time point as

$$
g_{s c}=\frac{E}{1.6\left(V P D L / P_{a t m}\right)} \text {. }
$$

$C_{\mathrm{i}}$ was then recalculated as

$$
C_{i}=C_{a}-\frac{A}{g_{s c}}
$$

Tests of the effect of gas exchange consistency were performed on the one-step methods only. 


\section{Results \\ Temporal estimates of absolute limitations}

Stomatal and biochemical components of $d A$ were calculated at each time point using the two elimination methods differing by the order in which limitations were removed, and the differential method. These were done for both the one-step and stepwise calculations of limitations

For the one-step calculation of limitations, the removal of stomatal limitation first led to estimates of predominantly biochemical limitation during the early stages of photosynthetic induction, with only a minimal stomatal limitation (Fig. 2a and Fig. 2b). In comparison, removal of the biochemical limitation first gave co-limitation between stomata and biochemistry of roughly the same magnitude in the early stages of photosynthetic induction (Fig. 2a and Fig. 2b). In both elimination methods the biochemical limitation rapidly decreased beyond the initial period of photosynthetic induction. However, the two elimination methods differed in the behaviour of stomatal limitation during photosynthetic induction: eliminating the biochemical limitation first produced a monotonically decreasing stomatal component of $d A$, while removing the stomatal limitation first gave a transiently rising stomatal component of $d A$, followed by a gradual decay in the stomatal component (Fig. 2a and Fig. 2b). The differential method produced stomatal and biochemical components of $d A$ with a similar temporal behaviour to the elimination method when the stomatal limitation was removed first, but in this example predicted a transiently higher stomatal limitation (Fig. 2c and 2d). The differential method also over-estimated $d A$ in the middle section of time courses when the stomatal opening time was slow and was caused by 
an over-estimation of $d A_{\text {stom }}$ (Fig. 2d). The mean of the two elimination methods on average followed the differential method better than the individual elimination methods when $d A_{\text {diff }}$ predicted $d A$ closely, except during the earliest stages of photosynthetic induction, when the mean predicted a higher stomatal limitation than the differential method (Fig. 2).

Using the stepwise calculation of limitations led to similar estimates of limitations for the differential method for fast stomatal opening and for the elimination method for slow stomatal opening, (Fig. 3c and 3b). However, there was less consistency in the estimate of limitations when using the elimination methods for fast stomatal opening, with an overestimation of the biochemical limitation when the stomatal limitation was removed first (Fig. 3a). The mean of the two elimination methods still produced limitations estimates similar to the differential method. The stepwise calculation of limitations using the differential method also led to near perfect calculation of $d A$ for the case of slow stomatal opening (Fig. 3d).

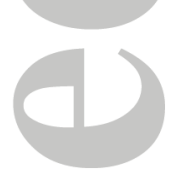

Time-integrated relative limitations during photosynthetic induction - comparison of elimination methods with the differential method

The time-integrated relative limitation predicted by elimination and differential methods provided a useful way to compare limitations among time courses of photosynthetic induction. Stomatal limitations estimated from the elimination methods were higher when the biochemical limitation was removed first and vice versa (Fig. 4). This was also apparent from inspection of the temporal dynamics of limitations, in particular during the early stages of photosynthetic induction (Fig. 2 and 3). 
Time-integrated relative limitations from the two elimination methods and their mean were compared with that from the differential method during photosynthetic induction. Using the one-step approach, removal of the biochemical limitation first tended to over-predict the total stomatal limitation compared to the differential method, while eliminating the stomatal limitation first led to the opposite occurrence, over the usual range of limitations between 0 and 1 (Fig. 4a and 4b). The mean of the two elimination methods, however, corresponded well with the differential method (Fig. 4b). A similar pattern was also observed using the stepwise calculation of limitations, except the over- and under-prediction of stomatal limitations by the elimination method compared with the differential method were greatly exacerbated (Fig 4c). Remarkably the mean of the two estimates from the elimination method again matched well with that predicted from the stepwise differential method (Fig. 4d).

Estimates of limitation estimates between one-step and stepwise differential methods were compared. The two methods compared well, except at stomatal limitations greater than 1 , where the one-step method under-predicted the limitation compared with the stepwise method (Fig 4e and Fig 4f).

Time-integrated relative limitations during photosynthetic induction - ranges and errors in the estimation of limitations

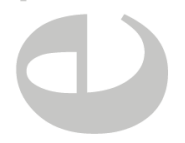

The reliability of the elimination and differential methods in calculating photosynthetic limitations was assessed by the relative range of the elimination method and the relative error of the differential method, respectively, for both one-step and stepwise calculation of limitations. Using relative range and error should remove the direct effect of different magnitudes of $A, g_{\mathrm{sc}}$ and $V_{\mathrm{cmax}}$ on the range and error. In doing so, the key variation should 
come from differences in the relative rates of biochemical activation ( $\left.t_{90 \mathrm{v}}\right)$ and stomatal opening $\left(t_{90 \mathrm{~g}}\right)$, measured here as durations.

Using the one-step calculation of limitations, relative range in the elimination method was largest when stomatal opening occurred over a timescale similar to that of biochemical activation, but reduced as stomatal opening became slower compared with biochemical activation (Fig. 5a). In contrast, relative error in the differential method was smallest for small values of $t_{90 \mathrm{~g}} / t_{90 \mathrm{~V}}$, that is biochemical activation and stomatal opening occurring at similar rates, but rapidly increased as stomatal opening became slower than biochemical activation (Fig. 5b).

Using the stepwise calculation of limitations, relative range in the elimination method was very large when stomatal opening and biochemical activation occurred over similar timescales, despite the simulated range being very small (Fig. 5c). However, at slow stomatal opening error was very small. Relative error in the differential method was predominantly small across the range of $t_{90 \mathrm{~g}} / t_{90 \mathrm{v}}$ for both observed data and simulations (Fig $5 \mathrm{~d}$ ). However it is worth noting that because the definition of relative range and error differs between the two methods, a direct, quantitative comparison should be avoided.

The effect of boundary layer conductance, ternary correction and consistency in gas exchange equations on limitation estimates

The effect of boundary layer conductance and the ternary correction on estimates of limitations was assessed by comparing the full limitation analyses including ternary and boundary layer conductance limitations against simpler models where both gas exchange data and limitation analyses assumptions were consistent. The comparisons were done for the onestep calculation of limitations only. Provided that the gas exchange data were calculated 
using similar assumptions to the analyses, predictions using the full and simpler analyses for the elimination method were similar (Fig. 6). However, when ternary corrected and boundary layer data were used in simpler analyses, limitation estimates from the elimination method were inconsistent (Fig. 6). In contrast, the differential method appeared immune from these effects and predicted limitations were largely consistent (Fig. 6).

\section{Discussion}

In response to an increase in irradiance, slow stomatal opening and activation of photosynthetic enzymes leads to plants forgoing photosynthesis compared with instantaneous photosynthetic induction (Deans et al., 2019; Kaiser et al., 2016; Kirschbaum \& Pearcy, 1988). Here we estimated the component of forgone photosynthesis corresponding to biochemical or stomatal limitations using two methods: the first sequentially eliminated the effect of each limitation, while the second calculated the component of each limitation using a tangent plane approximation. We did this at each time point by a one-step method that did not take into account the path of photosynthetic induction, and a stepwise method that used knowledge of future limitations to calculate the current one. We further calculated the contribution each limitation made to the total potential assimilation forgone and thus timeintegrated limitations during induction. Each method produces a different estimate for the component of each limitation, raising the question of which method is best to use and when.

We assessed how well each method estimated photosynthetic limitations during photosynthetic induction by considering either the relative range or the relative error of each method. We found that the differential method, when calculated in a stepwise manner, was 
the best method over a broad range of timescales of biochemical activation and stomatal opening (Fig. 5). This makes intuitive sense, as provided time steps during photosynthetic induction are small enough, then the stepwise differential method is akin to numerically integrating limitations during the induction.

The one-step differential method exhibited a range of relative error, from low when stomatal opening and biochemical activation occurred over similar timescales, to high when stomatal opening occurred slower than biochemical activation. Given plants tend to maintain the same $C_{\mathrm{i}}$ across different irradiances at steady state, stomatal opening and biochemical activation occurring over similar timescales is equivalent to $g_{\mathrm{sc}}$ and $V_{\mathrm{cmax}}$ changing proportionally such that $g_{\mathrm{sc}} / V_{\mathrm{cmax}}$ is the same throughout, or $C_{\mathrm{i}}$ constant. One can show analytically that the differential method predicts limitations between two states exactly when $g_{\text {sc }} / V_{\text {cmax }}$ is constant between the two states, or $C_{\mathrm{i}}$ constant, when $R_{\mathrm{d}}$ is ignored or small (for a proof, see the Supporting Information). This is because $A$ is a homogeneous function (of order 1) of both $g_{\mathrm{sc}}$ and $V_{\text {cmax }}$ (Farquhar, 1989). That is, if both $g_{\text {sc }}$ and $V_{\text {cmax }}$ are doubled, then $A$ is also doubled (assuming mesophyll conductance is infinite). This leads to $A$ behaving effectively linearly when $g_{\mathrm{sc}} / V_{\mathrm{cmax}}$ is constant, so that trajectories of constant $C_{\mathrm{i}}$ on the assimilation surface track linear paths across the surface, despite $A$ being a nonlinear function of $g_{\text {sc }}$ and $V_{\text {cmax }}$ separately (one such path can be visualised in Fig. 1d, when the tangent plane is always tangential to the "assimilation surface"). Thus, if it is known that $C_{\mathrm{i}}$ or $g_{\mathrm{sc}} / V_{\mathrm{cmax}}$ is constant between two states, then exact stomatal and biochemical limitations can be calculated. The equivalence of limitation sensitivity $(l)$ and relative limitation $(\sigma)$ when $C_{\mathrm{i}}$ is constant in Wilson et al. (2000) is also because of this homogeneity. Paths that are further away from constant $C_{\mathrm{i}}$ produce more curvature on the assimilation surface so that the one-step method becomes more error prone, but this is reduced in the stepwise method because slopes are recalculated at each new time point. 
How well the elimination methods estimated photosynthetic limitations was more difficult to assess. Here we used a metric, the relative range, that estimated how consistent estimates were between removing stomatal or biochemical limitations first. Although we simulated that the elimination method should be more consistent when calculated using the stepwise approach, the stepwise approach used on actual data was much less consistent than the onestep approach, other than when stomata were very slow to open. It appears that the elimination method when used in a stepwise fashion was too sensitive to small fluctuations in the gas exchange data. This could occur if over some time points largely different predictions in limitations were predicted for almost no change in assimilation because of noise.

The one-step method was also more consistent in limitation estimates when stomata were slower. This is equivalent to the change in photosynthesis between states being attributable to one limitation over the other and is in direct contrast to the differential method. The dichotomy regarding best method is fortunate for cases where the intermediate time points between states are not known, as it allows us to utilise the differential method when $g_{\mathrm{sc}} / V_{\mathrm{cmax}}$ or $C_{\mathrm{i}}$ is relatively constant, or to use the elimination method when $C_{\mathrm{i}}$ between the two states is different. This has implications for the calculation of limitations in general, beyond the use for photosynthetic induction. It is also interesting to note that the mean of the elimination methods typically produced limitation estimates that were similar to those predicted by the differential method, even when the individual elimination estimates were far from consistent. This is likely a consequence of the mean value theorem in mathematics.

Methods used to estimate biochemical and stomatal limitations to photosynthesis do not consider the effect of boundary layer conductance or the ternary correction, while also employing gas exchange data that do include their effect (Grassi \& Magnani, 2005; Wilson et $a l ., 2000)$. Inconsistencies between gas exchange data that do include the ternary correction and isotope equations that do not has previously been shown to produce errors (Farquhar \& 
Cernusak, 2012). Here we have shown that provided gas exchange data and limitation analyses are both calculated assuming consistent equations, then the effect of boundary conductance and the ternary correction is small for the elimination method. However, if the equations are inconsistent then significant errors in estimates of limitations can occur using the elimination method. In contrast, there was little effect of boundary layer conductance, the ternary correction or consistency of equations on estimates using the differential method. We therefore recommend in particular that if elimination methods are used then the gas exchange data should be calculated to be consistent with the equations used in the limitations analysis. However, if an adequate number of time points are known then using the differential method is highly encouraged, given the insensitivity of the method to consistency between equations used in the calculation of gas exchange and limitations.

We chose to define a limitation as the difference between two states - the current and the final state. This choice was intuitive for the question that we wanted to ask: of the difference in $A$ from the final $A$, or of total photosynthesis forgone, how much was due to stomatal or biochemical effects? We found that the best representation of temporal limitations during induction was by considering the absolute limitations directly. We also found that defining relative limitations in this way was also the most useful way to describe what proportion of total forgone photosynthesis was due to each limitation. Our definition of $\sigma$ is similar to that in Wilson et al. (2000). Defining limitations in this way was more intuitive than using a sensitivity limitation, $l$, (Grassi \& Magnani, 2005; Jones, 1985; Wilson et al., 2000). However, relative limitations defined as $l$ may be useful for considering the relative impact on $A$ for relative changes in stomatal or biochemical components. Limitations defined our way are also more realistic than defining limitations relative to completely removing limitations, that is letting $g_{\mathrm{sc}}$ or $V_{\mathrm{cmax}}$ be infinite, as described in Farquhar and Sharkey (1982). 


\section{Acknowledgements}

We thank the anonymous reviewers for helpful comments that allowed us to extend the scope of this work. This work was supported by the Australian Research Council Centre of Excellence for Translational Photosynthesis (CE1401000015). R.M.D. was supported by an ANU Gwendolyn Woodroofe PhD Scholarship.

\section{Conflict of Interest Statement}

The authors declare no conflict of interest.

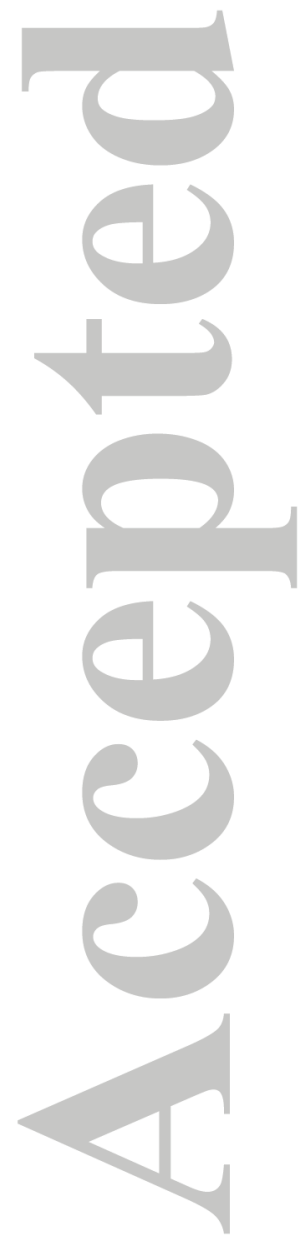




\section{References}

Assmann S.M. (1988) Stomatal and non-stomatal limitations to carbon assimilation: an evaluation of the path-dependent method. Plant, Cell \& Environment, 11, 577-582.

Busch F.A. \& Sage R.F. (2017) The sensitivity of photosynthesis to $\mathrm{O}_{2}$ and $\mathrm{CO}_{2}$ concentration identifies strong Rubisco control above the thermal optimum. New Phytologist, 213, 1036-1051.

Deans R.M., Brodribb T.J., Busch F.A. \& Farquhar G.D. (2019) Plant water-use strategy mediates stomatal effects on the light induction of photosynthesis. New Phytologist, 222, 382-395.

Douthe C., Dreyer E., Brendel O. \& Warren C.R. (2012) Is mesophyll conductance to $\mathrm{CO}_{2}$ in leaves of three Eucalyptus species sensitive to short-term changes of irradiance under ambient as well as low $\mathrm{O}_{2}$ ? Functional Plant Biology, 39, 435-448.

Douthe C., Dreyer E., Epron D. \& Warren C.R. (2011) Mesophyll conductance to $\mathrm{CO}_{2}$, assessed from online TDL-AS records of ${ }^{13} \mathrm{CO}_{2}$ discrimination, displays small but significant short-term responses to $\mathrm{CO}_{2}$ and irradiance in Eucalyptus seedlings. Journal of Experimental Botany, 62, 5335-5346.

Farquhar G.D. (1989) Models of integrated photosynthesis of cells and leaves. Philosophical Transactions of the Royal Society of London. B, Biological Sciences, 323, 357-367.

Farquhar G.D. \& Cernusak L.A. (2012) Ternary effects on the gas exchange of isotopologues of carbon dioxide. Plant, Cell \& Environment, 35, 1221-1231.

Farquhar G.D. \& Sharkey T.D. (1982) Stomatal conductance and photosynthesis. Annual Review of Plant Physiology, 33, 317-345.

Farquhar G.D., von Caemmerer S. \& Berry J.A. (1980) A biochemical model of photosynthetic $\mathrm{CO}_{2}$ assimilation in leaves of $\mathrm{C}_{3}$ species. Planta, 149, 78-90. 
Flexas J., Ribas-Carbo M., Diaz-Espejo A., Galmes J. \& Medrano H. (2008) Mesophyll conductance to $\mathrm{CO}_{2}$ : current knowledge and future prospects. Plant, Cell \& Environment, 31, 602-621.

Grassi G. \& Magnani F. (2005) Stomatal, mesophyll conductance and biochemical limitations to photosynthesis as affected by drought and leaf ontogeny in ash and oak trees. Plant, Cell \& Environment, 28, 834-849.

Hermida-Carrera C., Kapralov M.V. \& Galmés J. (2016) Rubisco catalytic properties and temperature response in crops. Plant Physiology, 171, 2549-2561.

Jarman P.D. (1974) The diffusion of carbon dioxide and water vapour through stomata. Journal of Experimental Botany, 25, 927-936.

Jones H.G. (1973) Limiting factors in photosynthesis. New Phytologist, 72, 1089-1094.

Jones H.G. (1985) Partitioning stomatal and non-stomatal limitations to photosynthesis. Plant, Cell \& Environment, 8, 95-104.

Kaiser E., Morales A. \& Harbinson J. (2018) Fluctuating light takes crop photosynthesis on a rollercoaster ride. Plant Physiology, 176, 977-989.

Kaiser E., Morales A., Harbinson J., Heuvelink E., Prinzenberg A.E. \& Marcelis L.F.M. (2016) Metabolic and diffusional limitations of photosynthesis in fluctuating irradiance in Arabidopsis thaliana. Scientific Reports, 6, 31252.

Kaiser E., Morales A., Harbinson J., Kromdijk J., Heuvelink E. \& Marcelis L.F. (2015) Dynamic photosynthesis in different environmental conditions. Journal of Experimental Botany, 66, 2415-2426.

Kirschbaum M.U.F. \& Pearcy R.W. (1988) Gas exchange analysis of the relative importance of stomatal and biochemical factors in photosynthetic induction in Alocasia macrorrhiza. Plant Physiology, 86, 782-785. 
Kromdijk J., Głowacka K., Leonelli L., Gabilly S.T., Iwai M., Niyogi K.K. \& Long S.P. (2016) Improving photosynthesis and crop productivity by accelerating recovery from photoprotection. Science, 354, 857-861.

Lawson T. \& Blatt M.R. (2014) Stomatal size, speed, and responsiveness impact on photosynthesis and water use efficiency. Plant Physiology, 164, 1556-1570.

McAusland L., Vialet-Chabrand S., Davey P., Baker N.R., Brendel O. \& Lawson T. (2016) Effects of kinetics of light-induced stomatal responses on photosynthesis and wateruse efficiency. New Phytologist, 211, 1209-1220.

Mott K.A. \& Woodrow I.E. (2000) Modelling the role of Rubisco activase in limiting nonsteady-state photosynthesis. Journal of Experimental Botany, 51, 399-406.

Slattery R.A., Walker B.J., Weber A.P. \& Ort D.R. (2018) The impacts of fluctuating light on crop performance. Plant Physiology, 176, 990-1003.

Taylor S.H. \& Long S.P. (2017) Slow induction of photosynthesis on shade to sun transitions in wheat may cost at least $21 \%$ of productivity. Philosophical Transactions of the Royal Society B: Biological Sciences, 372.

Tazoe Y., von Caemmerer S., Badger M.R. \& Evans J.R. (2009) Light and $\mathrm{CO}_{2}$ do not affect the mesophyll conductance to $\mathrm{CO}_{2}$ diffusion in wheat leaves. Journal of Experimental Botany, 60, 2291-2301.

Vico G., Manzoni S., Palmroth S. \& Katul G. (2011) Effects of stomatal delays on the economics of leaf gas exchange under intermittent light regimes. New Phytologist, 192, 640-652.

von Caemmerer S. \& Farquhar G.D. (1981) Some relationships between the biochemistry of photosynthesis and the gas exchange of leaves. Planta, 153, 376-387. 
Wilson K.B., Baldocchi D.D. \& Hanson P.J. (2000) Quantifying stomatal and non-stomatal limitations to carbon assimilation resulting from leaf aging and drought in mature deciduous tree species. Tree Physiology, 20, 787-797.

Wong S.C., Cowan I.R. \& Farquhar G.D. (1979) Stomatal conductance correlates with photosynthetic capacity. Nature, 282, 424-426.

Woodrow I.E. \& Berry J. (1988) Enzymatic regulation of photosynthetic $\mathrm{CO}_{2}$, fixation in $\mathrm{C}_{3}$ plants. Annual Review of Plant Physiology and Plant Molecular Biology, 39, 533-594.

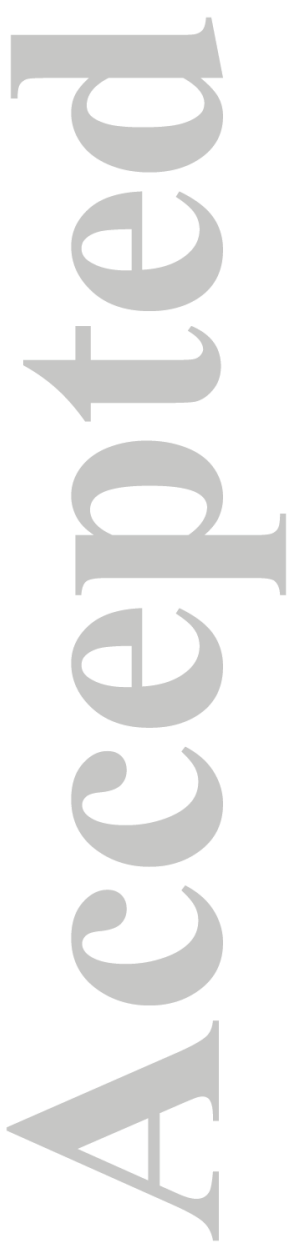



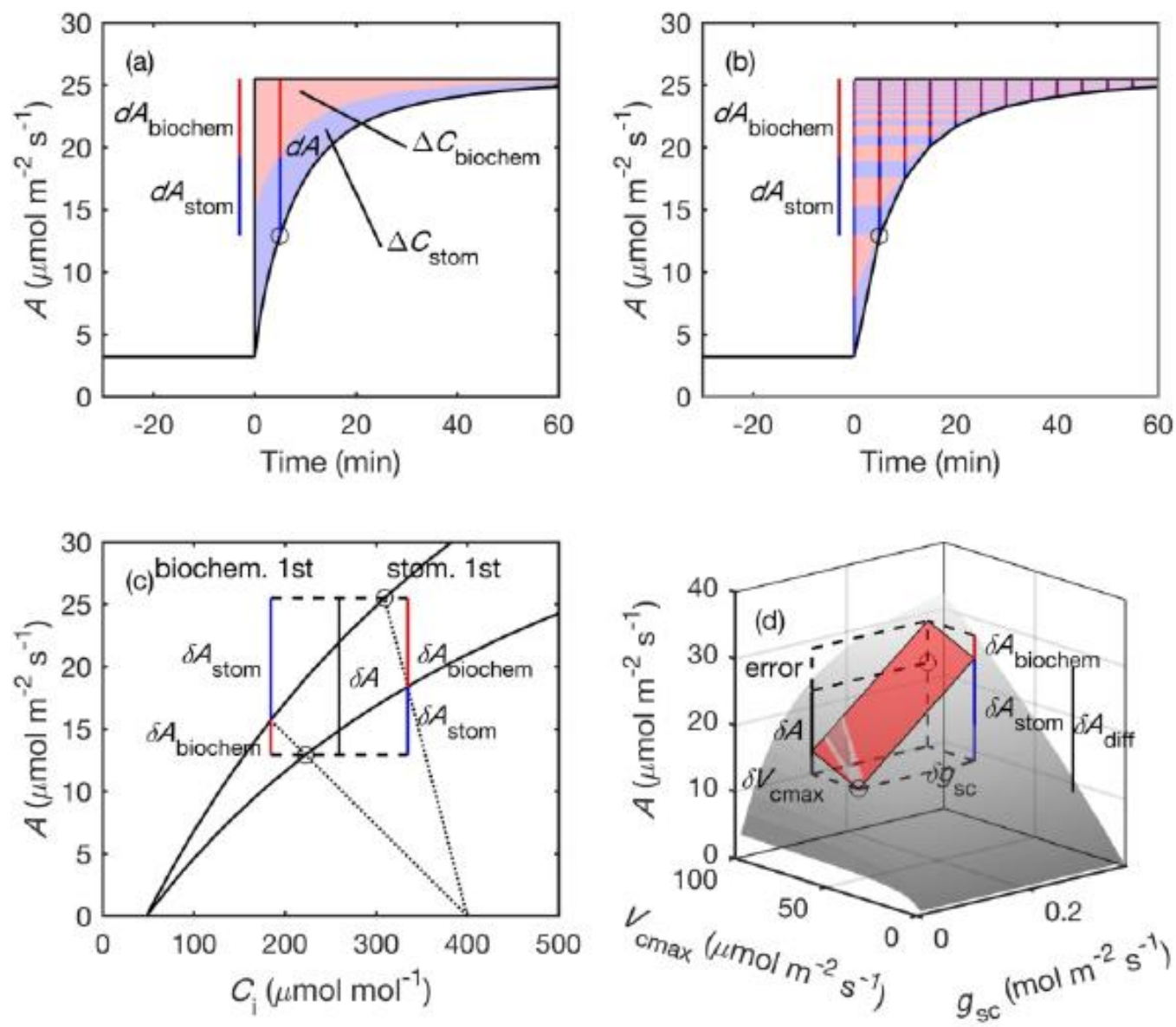

Figure 1. Methods and approaches to calculating stomatal and biochemical limitations during photosynthetic induction. (a) Calculating limitations using the one-step approach. Following an increase in irradiance at time $=0 \mathrm{~min}, A$ increases slowly due to delayed biochemical activation and stomatal opening, resulting in plants not achieving the potential assimilation rate that would occur if stomatal opening and biochemical activation had reached their final values instantaneously $(d A)$. Limitation analyses aim to separate this difference in photosynthesis into biochemical $\left(d A_{\text {biochem }}\right.$, red) and stomatal $\left(d A_{\text {stom }}\right.$, blue $)$ effects. In the onestep approach, limitations are calculated directly. Similarly they can also separate total assimilation forgone during induction (coloured area above curve) into biochemical

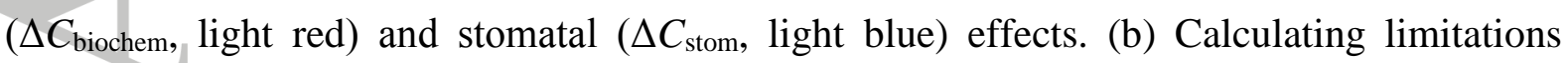
using the stepwise approach. This approach calculates limitations to the next time point. The 
limitation at the current time point is then the sum of all future limitation steps back to the current timepoint. (c) Calculation of limitations using the elimination methods, illustrated on an $A$ vs. $C_{\mathrm{i}}$ curve. The two black curves represent $A$ vs. $C_{\mathrm{i}}$ curves for $V_{\mathrm{cmax}}$ at the first (lower curve) and second (upper curve) photosynthetic state. Dotted lines represent $\mathrm{CO}_{2}$ supply curves, governed by the stomatal conductance in the first (lower line) and second (upper line) photosynthetic states. Removal of the biochemical limitation first (left side) involves tracking along the current $\mathrm{CO}_{2}$ supply line to the final $A$ vs. $C_{\mathrm{i}}$ curve, the difference in $A$ from the first state being $d A_{\text {biochem }}$ (red). $d A_{\text {stom }}$ (blue) is the remaining difference from the second $A$. Removal of the stomatal limitation first (right side) involves following the current $A$ vs. $C_{\mathrm{i}}$ curve until the second $\mathrm{CO}_{2}$ supply line is reached, the difference in $A$ from the first state being $d A_{\text {stom }}$ (blue). $d A_{\text {biochem }}$ (red) being the remaining difference from the final $A$. The estimates of biochemical and stomatal limitations are dependent on the order limitations are removed. (d) Calculation of limitations using the differential method. The light grey surface is the "assimilation surface" $A\left(V_{\mathrm{cmax}}, g_{\mathrm{sc}}\right)$. Open circles show the first and second photosynthetic states. A tangent plane (red plane) is projected tangential to the surface at the

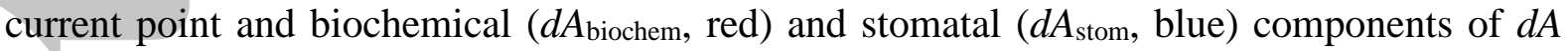
are constructed by traversing the plane between current and final $V_{\mathrm{cmax}}\left(d V_{\mathrm{cmax}}\right)$ and $g_{\mathrm{sc}}\left(d g_{\mathrm{sc}}\right)$, respectively, and calculating the difference in $A$ between these points and the first photosynthetic state. The sum of $d A_{\text {biochem }}$ and $d A_{\text {stom }}$ corresponds to the predicted difference in $A\left(d A_{\text {diff }}\right)$. The difference between the predicted and actual $d A$ is the error. 

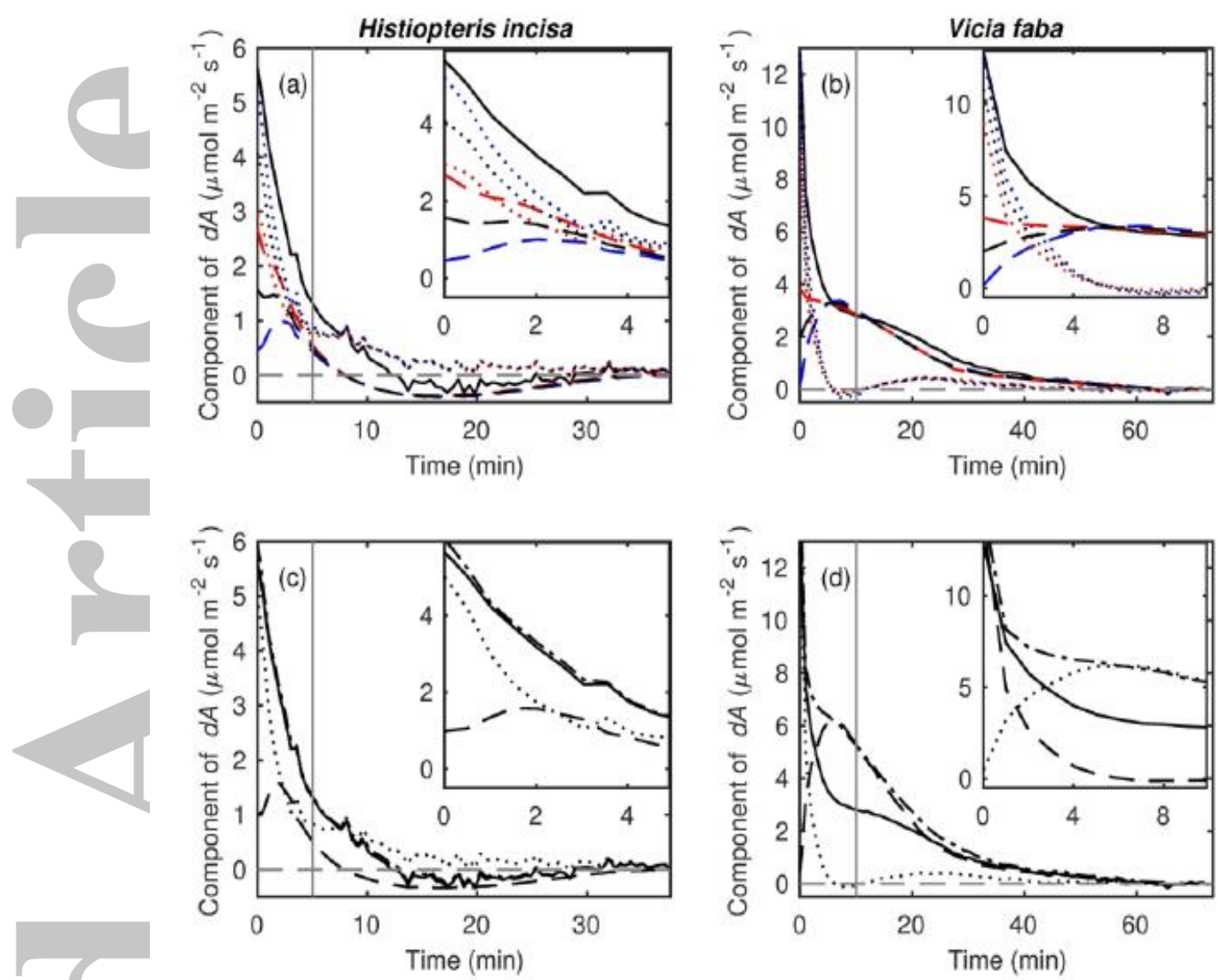

Figure 2. Two examples of the one-step calculation of components of $d A$ in Histiopteris incisa (a and c; fast stomatal opening) and Vicia faba (b and d; slow stomatal opening) using elimination ( $\mathrm{a}$ and $\mathrm{b}$ ) and differential ( $\mathrm{c}$ and d) methods. In all figures the solid black line is the instantaneous difference in photosynthetic rate $(d A)$. In elimination methods ( $\mathrm{a}$ and $\mathrm{b}$ ), Stomatal limitations are shown as dashed lines and biochemical limitations as dotted lines. Red lines represent limitations calculated by removing the biochemical limitation first, blue lines represent removing the stomatal limitation first, and black lines represent the mean of the two. In the differential method (c and d), dashed lines represent stomatal limitations, dotted lines represent biochemical limitations and the dotted-dashed line represents $d A$ estimated from the differential method $\left(d A_{\text {diff }}\right)$. The initial part of photosynthetic induction is shown in insets. Horizontal grey dashed lines show where $d A$ is zero, vertical solid grey lines show the maximum time cut-off shown in the insets. 

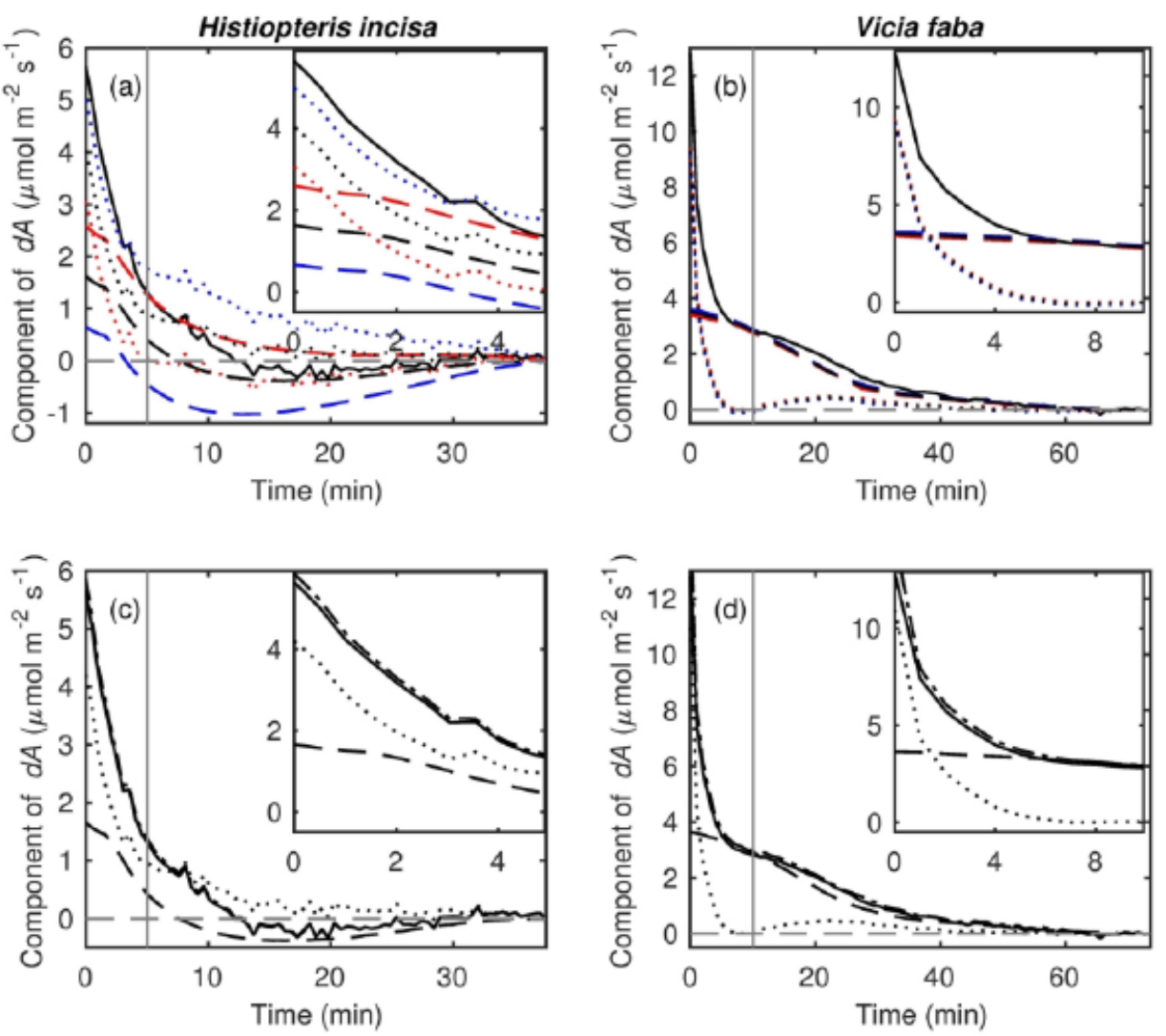

Figure 3. Two examples of the stepwise calculation of components of $d A$ in Histiopteris incisa (a and c; fast stomatal opening) and Vicia faba (b and d; slow stomatal opening) using elimination ( $\mathrm{a}$ and $\mathrm{b}$ ) and differential ( $\mathrm{c}$ and $\mathrm{d}$ ) methods. In all figures the solid black line is the instantaneous difference in photosynthetic rate $(d A)$. In elimination methods ( $\mathrm{a}$ and $\mathrm{b}$ ), stomatal limitations are shown as dashed lines, biochemical limitations as dotted lines. Red lines represent limitations calculated by removing the biochemical limitation first, Blue lines represent removing the stomatal limitation first, and black lines represent the mean of the two. In the differential method (c and d), dashed lines represent stomatal limitations, dotted lines represent biochemical limitations and the dotted-dashed line represents $d A$ estimated from the differential method ( $\left.d A_{\text {diff }}\right)$. The initial part of photosynthetic induction is shown in insets. Horizontal grey dashed lines show where $d A$ is zero, vertical solid grey lines show the maximum time cut-off shown in the insets. 

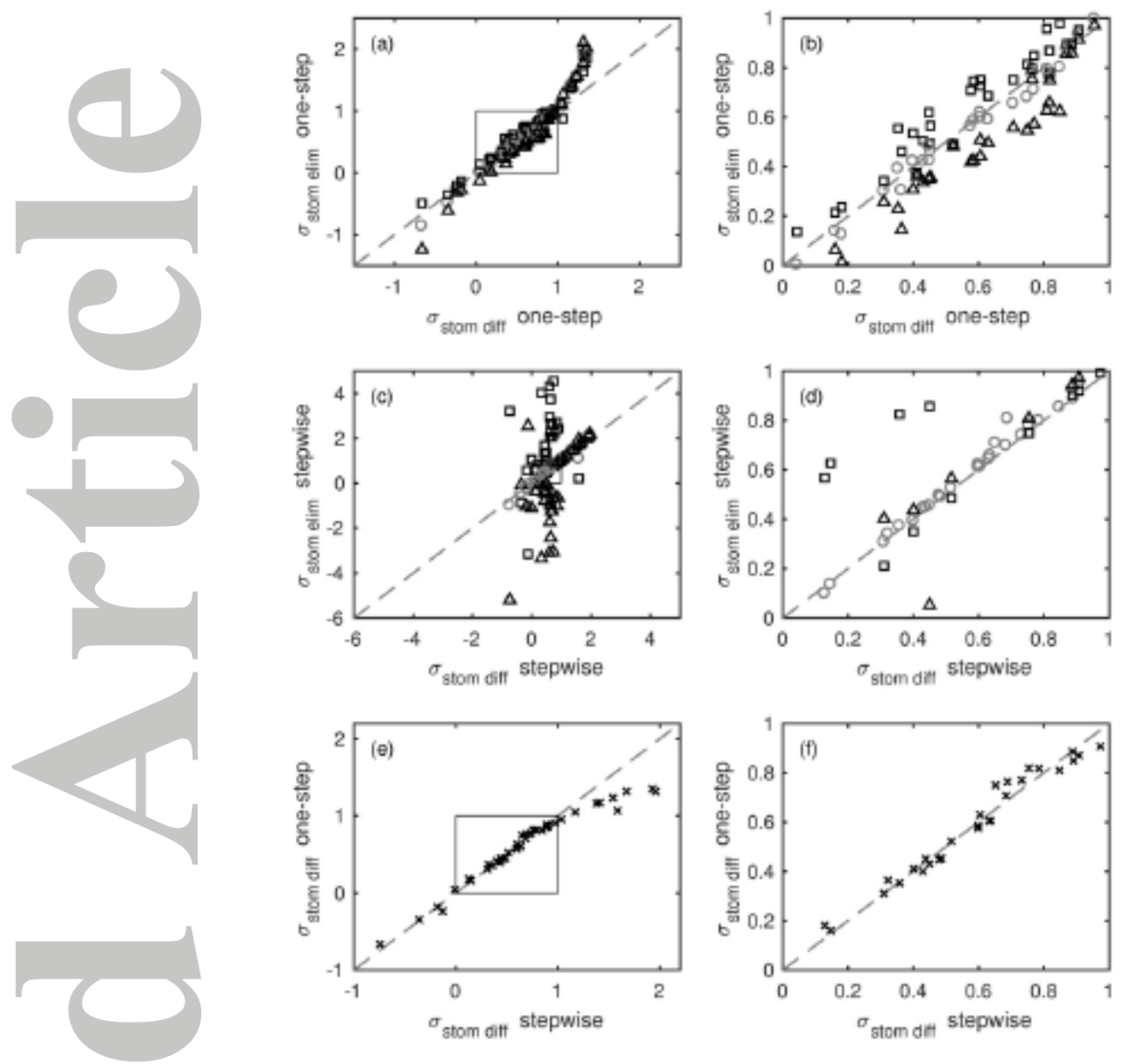

Figure 4. Comparison between the time-integrated relative stomatal limitations calculated during photosynthetic induction. (a) and (b) Comparison of stomatal limitations $\left(\sigma_{\text {stom }}\right)$ between the elimination and differential methods using the one-step approach. (c) and (d) Comparison of stomatal limitations between the elimination and differential methods using the stepwise approach. (e) and (f) Comparison of stomatal limitations from the differential method, calculated by the one-step and stepwise approaches. Black squares represent limitations calculated by removing the biochemical limitation first, black triangles represent the removal of stomatal limitation first and grey circles represent the mean of the two. (b), (d) and (f) are insets of (a), (c) and (e) around the usual limitation range between 0 and 1 . The grey dashed line is $1: 1$. 

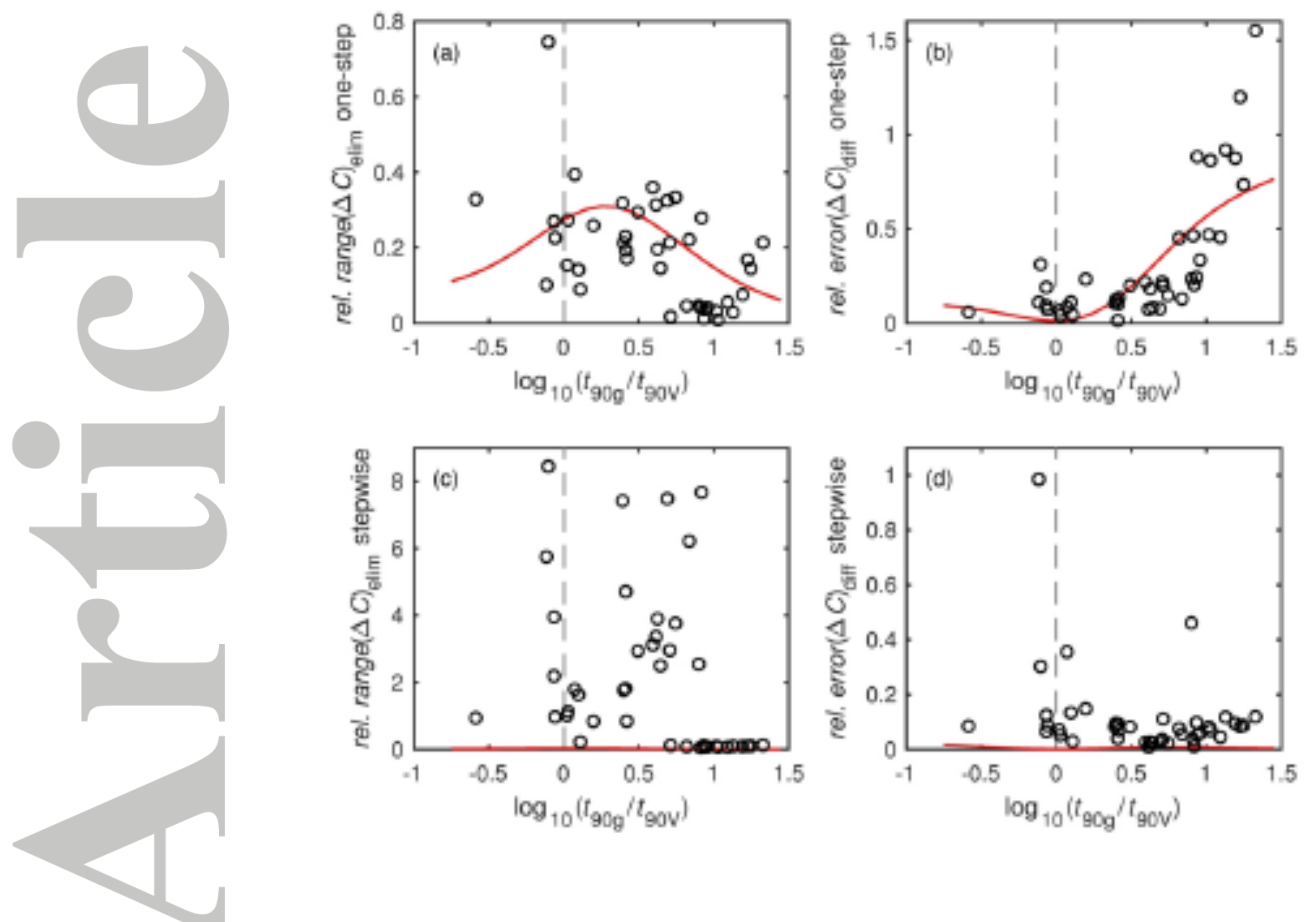

Figure 5. Predicted and observed relative range of the elimination methods and relative error of the differential method, for both one-step and stepwise approaches. (a) Relative range in forgone photosynthesis for the elimination method (rel. range $\left.(\Delta C)_{\text {elim}}\right)$ using the one-step approach, (b) relative error for the differential method (rel. error $\left.(\Delta C)_{\text {diff }}\right)$ using the one-step approach, (c) relative range for the elimination method using the stepwise approach and (d) relative error for the differential method (rel. error $\left.(\Delta C)_{\text {diff }}\right)$ using the stepwise approach, all compared with the ratio of stomatal opening to biochemical activation times ( $\left.t_{90 \mathrm{~g}} / t_{90 \mathrm{~V}}\right)$. Open black circles represent actual data and solid red lines represent modelled relative range or error assuming exponential biochemical and stomatal kinetics and constant internal $\mathrm{CO}_{2}$ concentration $\left(C_{\mathrm{i}}\right)$ at initial and final states. Grey dashed lines show when biochemical activation and stomatal opening occur at the same rate.

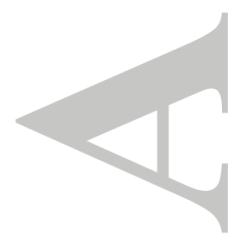



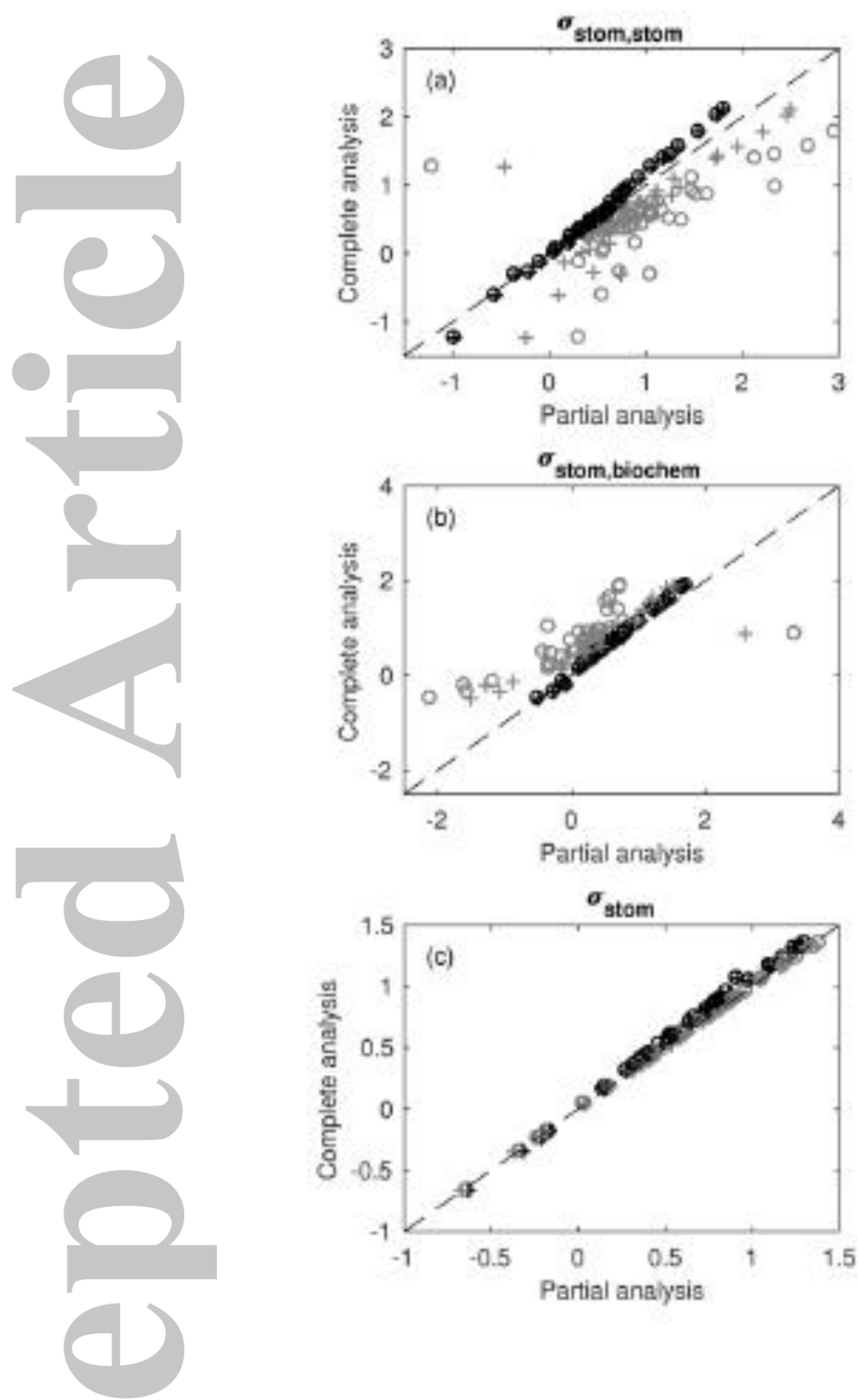

Figure 6. Comparisons of stomatal limitation estimates with different gas exchange equations. Complete analysis refers to the relevant limitation analysis with both ternary correction and boundary layer conductance equations, used on data that were calculated also with the ternary correction and boundary layer conductance. Partial analyses are limitation analyses performed using simpler gas exchange equations, on data that either were calculated using the ternary correction and boundary layer conductance (grey) or data that were 
recalculated to match the gas exchange equations in the analysis (black). Simpler analyses were: no ternary correction and no boundary layer conductance (open circle), and no ternary correction but including boundary layer conductance (plus signs). (a) and (b) are elimination methods with: (a) stomatal limitation removing stomata first $\left(\sigma_{\text {stom,stom }}\right)$ and (b) stomatal limitation removing biochemistry first $\left(\sigma_{\text {stom,biochem}}\right)$. (d) Stomatal limitations calculated by the differential method $\left(\sigma_{\text {stom }}\right)$. Grey dashed lines are 1:1 lines.

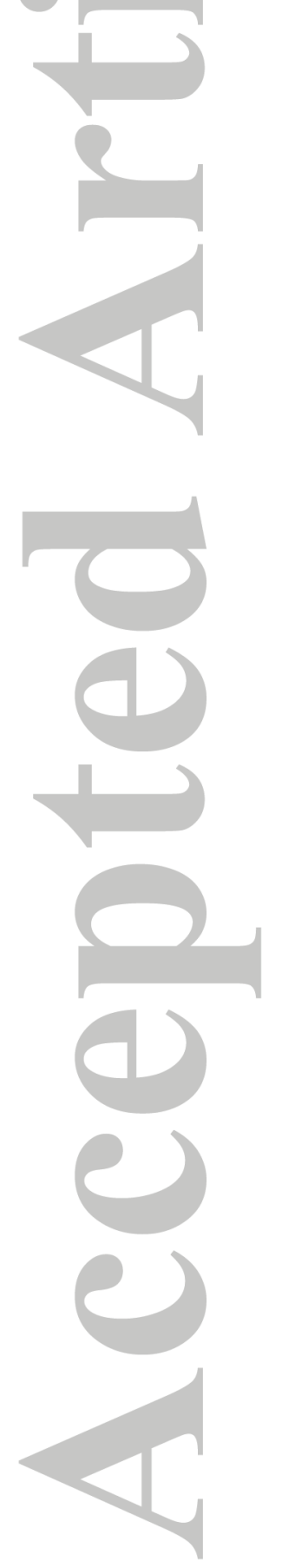

